\title{
A Framework for the Analysis of Majority Voting
}

\author{
Anand M. Narasimhamurthy \\ Department of Computer Science and Engineering \\ The Pennsylvania State University \\ University Park, PA-16802 \\ narasimh@cse.psu.edu
}

\begin{abstract}
Majority voting is a very popular combination scheme both because of its simplicity and its performance on real data. A number of earlier studies have attempted a theoretical analysis of majority voting. Many of them assume independence of the classifiers while deriving analytical expressions. We propose a framework which does not incorporate any assumptions. For a binary classification problem, given the accuracies of the classifiers in the team, the theoretical upper and lower bounds for performance obtained by combining them through majority voting are shown to be solutions of a linear programming problem. The framework is general and could provide insight into majority voting.
\end{abstract}

\section{Introduction}

The performance of majority voting has been demonstrated experimentally in a number of studies such as handwriting recognition [10,3], person authentication [4] and so on. A simple analytical justification for majority voting may be given by the well known Condorcet's theorem ( [2], [1]). Under the assumption of independent classifiers, if the individual classifier error rate $p<0.5$ (assume for simplicity that all classifiers have the same error rate), for odd number of classifiers(v oters) $N$, the correct decision rate increases with increasing $N$. A number of studies have addressed the problem of a theoretical analysis of majority voting $[4,8,7,6,5]$. Some of them analysed majority voting under the assumption that the classifiers are independent [8]. In [9] Miller and Yan describe a critic-driven ensemble classification scheme and provide an analysis of their scheme for the case of dependent voters, under the assumption that all the experts (voters/classifiers) are statistically identical. Some of the more recent work $[7,5]$ are aimed at providing insights based on pairwise dependence statistics since majority vote with dependent classifiers can offer improvement over independent classifiers and over the individual accuracies.

This paper is organised as follows. In section 2 the problem is formulated as a linear programming problem. For a binary classification problem, the bounds of performance of majority voting for a group of classifiers with known accuracies may be obtained as solutions to the linear programs. To the best of our knowledge we are not aware of any work which finds the theoretical bounds of majority voting under no assumptions, although some of the more recent work address the performance empirically $[7,5]$. Conclusions are presented in section 3. 


\subsection{Notation and Representation}

We restrict ourselves to a binary classification problem with no reject option (for the individual classifiers) for the rest of the paper. In this paper we represent the joint statistics (dependencies) through Venn diagrams. Figure 1 represents the joint statistics for 3 classifiers. In the Venn diagram the regions are numbered as follows. Each classifier is represented by a bit ( 1 or 0$)$ with 1 indicating that the classifier is correct and 0 indicating that the classifier is incorrect. The regions correspond to bit combinations. We follow the convention that if there are $\mathrm{n}$ classifiers $C 1, C 2, \ldots, C n, \mathrm{C} 1$ is the LSB (least significant bit) and Cn corresponds to the MSB (most significant bit). For example, in figure 1 region 5 corresponds to the bit combination 101 i.e. it corresponds to the event ( $\mathrm{C} 3$ is correct, $\mathrm{C} 2$ is wrong, $\mathrm{C} 1$ is correct) and the numbers in the regions indicate the probabilities associated the specific regions (events). For example, $x_{5}=\mathrm{P}($ Event represented by region 5$)=\mathrm{P}(\mathrm{C} 3$ correct, $\mathrm{C} 2$ incorrect, $\mathrm{C} 1$ correct $)$.

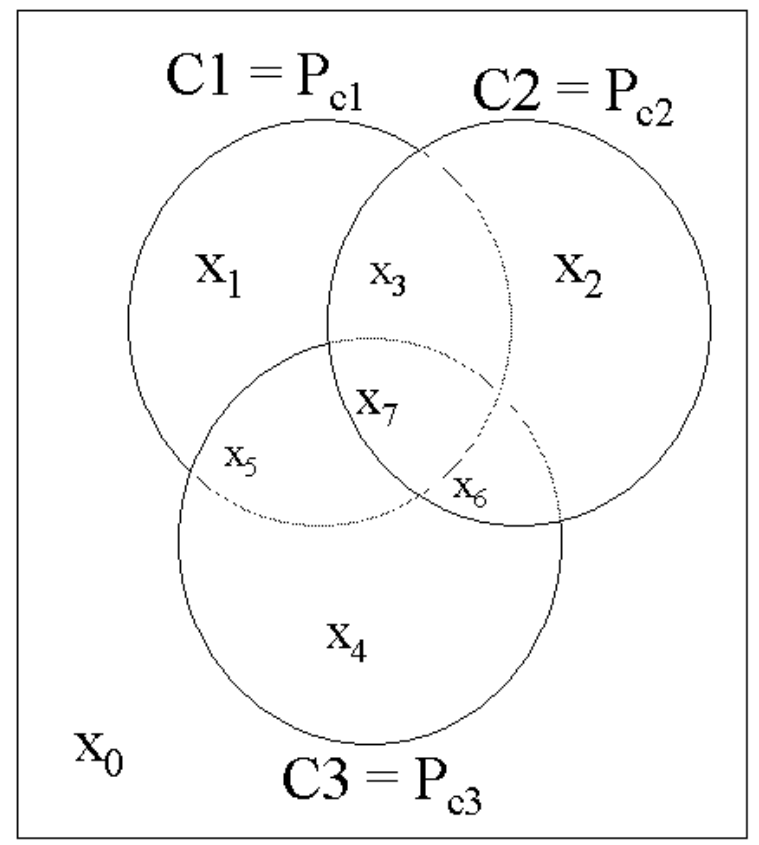

Fig. 1. Venn diagram showing joint statistics of 3 classifiers.

\section{Problem Formulation}

In this section we derive the theoretical upper and lower bounds of majority voting performance for a two class problem, given a set of classifiers whose accuracies are known. 
Specifically we address the following problem :

"For a binary classification problem, given a set of $K$ classifiers with known accuracies $P_{c 1}, \ldots, P_{c K}$ respectively, what are the theoretical upper and lower bounds for the majority voting scheme which combines the outputs of the individual classifiers."

These bounds are shown to be solutions of a linear programming problem (LP). The LP for $\mathrm{K}$ classifiers (K odd) is given by equations ( 1) - (3).

$$
\max (\min ) f_{m a j}^{T} \boldsymbol{x}
$$

s.t.

$$
\begin{gathered}
A_{e q} \boldsymbol{x}=p \\
\mathbf{0} \leq \boldsymbol{x} \leq 1 \\
\mathbf{1}^{T} \boldsymbol{x}=1
\end{gathered}
$$

where,

$$
\begin{aligned}
p & =\left[P_{c 1}, P_{c 2}, \ldots P_{c K}\right]^{T} \\
x & =\left[x_{0}, x_{1}, x_{2}, \ldots, x_{\left(2^{K}-1\right)}\right]^{T} \\
\text { and } A_{e q} & =\left[b_{1}, b_{2}, \ldots, b_{K}\right]^{T}
\end{aligned}
$$

$b_{1}, b_{2}, \ldots, b_{K}$ are as in equation 4 and $f_{m a j}$ is as in equation 5 .

We note that the vectors $f_{m a j}, b_{1}, \ldots b_{K}$ are infact bit strings of length $\mathrm{K}$ i.e. each component can be either a 1 or a 0 . For $b_{1}, b_{2}, \ldots, b_{K}$, the $i$ th bit corresponds to the $i t h$ classifier. It is a 1 if the classifier is correct and 0 otherwise. For example, $\frac{T_{1}}{x}=P_{c 1}$ essentially means the the sum of probabilities in all regions where classifier 1 is correct, is $P_{c 1}$. It can be easily seen that,

$$
\begin{aligned}
& b_{1}=\left[\begin{array}{llll}
0 & 1, \ldots, 0 & 1
\end{array}\right]^{T} \\
& b_{2}=\left[\begin{array}{llllllll}
0 & 0 & 1 & 1, \ldots, & 0 & 0 & 1 & 1
\end{array}\right]^{T} \\
& b_{K}=[\overbrace{00 \ldots 00}^{2^{(K-1)}}, \overbrace{11 \ldots 11}^{2^{(K-1)}}]^{T}
\end{aligned}
$$

Let $\operatorname{bit}(i, K)$ denote the $K$ bit binary representation of integer $i$. Then,

$$
f_{m a j}(i)=1 \text { iff number of } 1 \mathrm{~s} \text { in } \operatorname{bit}(i, K)>K / 2
$$

This corresponds to the fact that the Majority vote is right in a region (and hence the corresponding bit is a 1) where a majority of the classifiers are right. 
We draw the reader's attention to [5] Table III in which the authors analyze the case of 3 classifiers with accuracies of 0.6 each by considering 10 examples and building a table of all possibilities where each classifier classifies exactly 6 examples. The lower and upper bounds of majority voting in the table are 0.4 and 0.9 respectively. It can be verified by solving the linear program given by equation 1 and constraints in 2 with $\mathrm{Pc} 1=\mathrm{Pc} 2=\mathrm{Pc} 3=0.6$, that these are indeed the theoretical bounds in this case.

The theoretical upper and lower bounds of majority voting for 3 and 5 classifiers are illustrated in figures 2 (a) (3 classifiers) and 2(b) (5 classifiers). Here $p$ represents the accuracy of a single classifier in the pool and for purposes of illustration, all classifiers are assumed to have the same accuracy. $p$ was varied from 0 to 1 in steps of 0.05 and the corresponding lower and upper bounds of majority voting $\left(s_{\min }^{(M V)}(p)\right.$ and $\left.s_{\max }^{(M V)}(p)\right)$ were determined for each value of $p$. The curve inside the region bounded by the upper and lower bounds represents the majority vote accuracy if classifiers independent $\left(s_{\text {indep }}^{(M V)}(p)\right)$. Taking the Bayes accuracy (which is inherently related to the problem) into account, the bounds need to be modified as :

$$
\begin{aligned}
& s_{\min }^{(M V)}(p)=\min \left(s_{\min }(p), p_{\text {Bayes }}\right) \\
& s_{\max }^{(M V)}(p)=\min \left(s_{\max }(p), p_{\text {Bayes }}\right)
\end{aligned}
$$

where,

$s_{\min }(p), s_{\max }(p)$ are the solutions of the corresponding LPs and $p_{\text {Bayes }}$ is the Bayes accuracy (i.e. 1 - Bayes error rate). It is observed that the difference between the upper and lower bounds decreases for increasing $p$ indicating that higher the competence of the experts in the pool better is the worst case scenario.

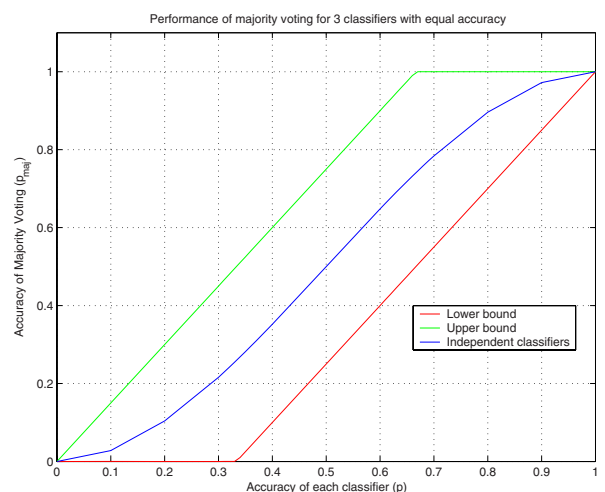

(a)

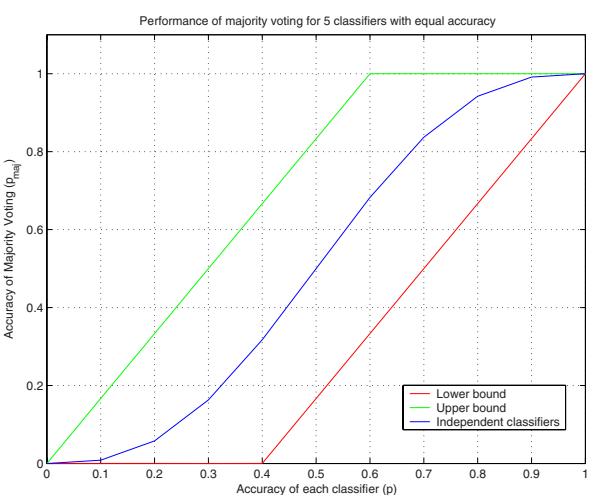

(b)

Fig. 2. Majority voting accuracy : Lower,upper bounds and independent classifiers for (a) 3 Classifiers (b) 5 classifiers with equal accuracy 
We state and prove the following proposition.

Proposition 1 Let $s_{\min }(\boldsymbol{p})$ and $s_{\max }(\boldsymbol{p})$ represent the lower and upper bounds of majority voting i.e. solutions of the LP 1 with the objective minimized and maximized respectively where $p=\left[P_{c 1}, P_{c 2}, \ldots P_{c K}\right]^{T}$ where $K$ is odd. Then,

$$
s_{\min }(\boldsymbol{p})+s_{\max }(\mathbf{1}-\boldsymbol{p})=1
$$

For the proof of the proposition, we need to prove two properties of the vectors $f_{m a j}, b_{1}, \ldots b_{K}$. Let $b$ be a bit string and let $b(i)$ denote the bit in the $i$ th position of $b$, let $\bar{b}$ denote the 1 s complement and let $b^{c}$ denote a bit string obtained by switching bit at position $i$ with bit at position $2^{K}-1-i$, i.e. $b^{c}(i)=b\left(2^{K}-1-i\right)$.

\section{Property 1}

$$
1-b^{T} \boldsymbol{x}=\bar{b}^{T} \boldsymbol{x}
$$

Proof : Since $\mathbf{1}^{T} \boldsymbol{x}=1$, we have, $1-b^{T} \boldsymbol{x}=\mathbf{1}^{T} \boldsymbol{x}-b^{T} \boldsymbol{x}=(\mathbf{1}-b)^{T} \boldsymbol{x}=\bar{b}^{T} \boldsymbol{x}$

\section{Property 2}

$$
\left(b^{c}\right)^{T} x=\bar{b}^{T} x
$$

Proof : From equation 4 it can be verified that all the vectors (bit strings) $b_{1}, b_{2}, \ldots b_{K}$ satisfy Property 2. For $f_{m a j}$ noting that if number of $1 \mathrm{~s}$ in $\operatorname{bit}(i, K)>K / 2$ then number of $1 \mathrm{~s}$ in $\operatorname{bit}\left(2^{K}-i-1, K\right)<K / 2$, since $2^{K}-i-1$ is in fact the 1 s complement of $i$, we have,

if $f_{m a j}(i)=1$ then $f_{m a j}\left(2^{K}-i-1\right)=0$.

Note that in the case of odd $K$ the number of 1 s and the number of 0 s is the same.

Hence for odd $K$ we have,

$$
\left(f_{m a j}^{c}\right)^{T} \boldsymbol{x}=f_{m a j}^{-}{ }^{T} \boldsymbol{x}
$$

Proof of proposition 1 :

Let LP1 denote the Linear program specified by equation 1 and constraints 2 . Let $s_{\min }(\boldsymbol{p})=\min f_{m a j}^{T} \boldsymbol{x}$ be the solution of LP1. By properties 1 and 2, we have

$$
\left(f_{m a j}^{c}\right)^{T} \boldsymbol{x}=1-f_{m a j}^{T} \boldsymbol{x}
$$

and thus

$$
\max \left(f_{m a j}^{c}\right)^{T} x=1-\min f_{m a j}^{T} x
$$


Consider the Linear program LP2 where the objective is max $\left(f_{m a j}^{c}\right)^{T} \boldsymbol{x}$ with the same set of constraints as LP1 (specified by constraints 2). Taking 1 - both sides, for all constraints we get linear program LP2 as :

$$
\max \left(f_{m a j}^{c}\right)^{T} \boldsymbol{x}
$$

s.t.

$$
\begin{gathered}
\left(b_{1}^{c}\right)^{T} x=1-P_{c 1} \\
\vdots \\
\left(b_{K}^{c}\right)^{T} x=1-P_{c K} \\
0 \leq x \leq 1 \\
\mathbf{1}^{T} x=1
\end{gathered}
$$

It can be seen that Linear Program LP2 is exactly equivalent to Linear Program LP3 where the objective function is given by equation 12 and the constraints by 13

$$
\begin{array}{cc}
\max & f_{m a j}^{T} x \\
\text { s.t. } & b_{1}^{T} x=1-P_{c 1} \\
\vdots \\
\\
b_{K}^{T} x=1-P_{c K} \\
\mathbf{0} \leq x \leq 1 \\
\mathbf{1}^{T} x=1
\end{array}
$$

This proves the proposition.

\section{Conclusions}

In this paper we formulate the problem of finding the lower and upper bounds of majority vote performance for a binary classification problem, as a linear programming (LP) problem. The lower and upper bounds are solutions of the corresponding LPs. No assumptions of independence of classifiers is made. It is hoped that this framework could be useful in providing insights into majority voting.

\section{Acknowledgement.}

The author expresses his sincere thanks to Prof. Rangachar Kasturi for valuable suggestions. 


\section{References}

1. S. Berg. Condorcet's jury theorem,dependency among jurors. Social Choice Welfare, 10:8795, 1993.

2. P. J. Boland. Majority systems and the condorcet jury theorem. Statistician, 38:181-189, 1989.

3. Tin Kam Ho, Jonathan J. Hull, and Sargur N. Srihari. Decision combination in multiple classifier systems. IEEE Transactions on Pattern Analysis and Machine Intelligence, 16(1):6675, January 1994.

4. Josef Kittler, Mohamad Hatef, Robert P. W. Duin, and Jiri Matas. On combining classifiers. IEEE Transactions on Pattern Analysis and Machine Intelligence, 20(3):226-239, March 1998.

5. L. I. Kuncheva, C. J. Whitaker, C.A. Shipp, and R.P.W. Duin. Limits on the majority vote accuracy in classifier fusion. Pattern Analysis and Applications.

6. Ludmila I. Kuncheva. A theoretical study on six classifier fusion strategies. IEEE Transactions on Pattern Analysis and Machine Intelligence, 24(2):281-286, February 2002.

7. Ludmila I. Kuncheva and C. J. Whitaker. Measures of diversity in classifier ensembles and their relationship with the ensemble accuracy. Machine Learning, 51:181-207, 2003.

8. L. Lam and C.Y. Suen. Application of majority voting to pattern recognition: An analysis of its behavior and performance. IEEE Transactions on Systems, Man and Cybernetics, 27(5):553-568, 1997.

9. David J. Miller and Lian Yan. Critic-driven ensemble classification. IEEE Transactions on Signal Processing, 47(10):2833-2844, October 1999.

10. L. Xu, A. Krzyzak, and C.Y. Suen. Methods of combining multiple classifiers and their application to handwriting recognition. IEEE Transactions on Systems, Man and Cybernetics, 22:418-435, 1992. 